\title{
Alteration of Coagulation Test Results and Vaginal Bleeding Associated With the Use of Feverfew (Tanacetum parthenium)
}

\author{
Khalidah A. Alenzi ${ }^{a}$, c , Fasil H. Alharbia ${ }^{a}$, Fasil M. Tawharia ${ }^{\text {a }}$, Ghada S. Fradees ${ }^{b}$
}

\begin{abstract}
Tanacetum parthenium (feverfew) is a member of the daisy family; it is used to prevent and treat migraine and rheumatoid arthritis. It has a long history of use as a traditional and folk medicine in Chinese, Greek, Indian and Arabic medicine, having been used for hundreds of years. The term feverfew comes from the Latin word febrifugia and means fever reducer. However, Short term use of feverfew (up to 4 months) is considered safe in adults. According to a few clinical trials, Tanacetum parthenium was not associated with serious adverse events but rather with mild and reversible events. Adverse events leading to withdrawals were mainly of a gastrointestinal nature. There is no major safety issue. Nevertheless, we report one case of a 36-year-old woman with known migraine who visited the obstetrics and gynecology clinic upon developing vaginal bleeding, prolonged duration of the menstrual cycle, and reddish skin without bruising. The patient suffered from these symptoms over a period of 3 months prior to the clinic visit. Based on history, the patient began taking $800 \mathrm{mg}$ capsules of feverfew three times per day 9 months ago. We applied the Naranjo scale in our case, and it indicated that a probable relationship exists between feverfew and vaginal bleeding. Feverfew should be used cautiously by patients planning elective surgery, having coagulant disorders or taking antithrombotic drugs.
\end{abstract}

Keywords: Tanacetum parthenium; Feverfew; Vaginal bleeding; Migraine; Naranjo adverse drug reaction probability scale

\section{Introduction}

Tanacetum parthenium has an interesting history. It is a mem-

Manuscript submitted October 6, 2020, accepted October 17, 2020

Published online November 18, 2020

${ }^{a}$ Ministry of Health, Regional Drug Information and Pharmacovigilance Center, Tabuk, Saudi Arabia

bMinistry of Health, Prince Sultan Cardiac Center, Al-Qassim, Saudi Arabia ${ }^{\mathrm{c} C}$ Corresponding Author: Khalidah A. Alenzi, Regional Drug Information and Pharmacovigilance Center, General Directorate of Health Affairs in Tabuk Region, P.O. Box 876 King Fahad Hospital, Tabuk, Saudi Arabia.

Email:ph_kh@hotmail.com

doi: https://doi.org/10.14740/jmc3601 ber of the daisy family, and the plant originated in Europe, North America and Australia. According to a few clinical trials, Tanacetum parthenium was not associated with serious adverse events but rather with mild and reversible events [1]. Adverse events leading to withdrawals were mainly of a gastrointestinal nature [1]. There is no major safety issue. It is mentioned by common names, such as feverfew and featherfew $[2,3]$.

In the past, feverfew was used by Greeks and Europeans to treat fevers, migraine headaches and bites [2, 4]. In 1978, it was first freely used as a preventative for migraine in the UK. Many clinical trials support the use of feverfew to prevent migraine and treat rheumatic disease, arthritis and irregular menstrual cycles $[2,5]$.

The medicinal components of feverfew are its leaves and the flower heads, and the constituents of the plant are a volatile oil, flavonoids, polyenes and sesquiterpene lactones $[5,6]$. More than 30 sesquiterpene lactones have been identified in feverfew. Sesquiterpene lactones are active constituents in feverfew, especially parthenolide, 3-beta-hydroxyparthenolide, tanaparthin-alpha-peroxide, costunolide, reynosin, 8-beta hydroxyreynosinn, canin, artecanin and secotanaparthenolide A $[5,6]$. The leaves are cut before complete flowering and dried at temperatures not exceeding $35{ }^{\circ} \mathrm{C}[2,7]$. Many commercial feverfew preparations are standardized based on a $0.1 \%$ to $0.2 \%$ parthenolide content $[2,8]$.

The Few Gs are herbs that have been associated with an increased risk of bleeding or having coumarin-like or salicylate-like effects; the name Few Gs was proposed by Ciocon and other writers to help health care professionals remember a list of herbs that are known to alter bleeding. The Few Gs include feverfew, ginger, ginkgo biloba, garlic and ginseng $[9,10]$.

Possible adverse drug reactions associated with feverfew use are abdominal pain, nausea, vomiting, mouth sores and increased risk of bleeding. Stopping feverfew therapy can lead to withdrawal symptoms such as insomnia, joint pain, nervousness and headache, and patients should be advised to taper then discontinue feverfew completely 2 - 3 weeks before surgery or when starting any anticoagulant or platelet therapy $[11,12]$.

\section{Case Report}

A 36-year-old female visited the obstetrics and gynecology clinic with complaints of vaginal bleeding, a prolonged dura- 
Table 1. Laboratory Results

\begin{tabular}{llll}
\hline Test & First test & After 4 months & Normal range \\
\hline Prothrombin time (s) & 27.3 & 14.4 & $11-16$ \\
Partial thromboplastin time (s) & 42 & 29 & $18-28$ \\
Hemoglobin (g/dL) & 10 & 12 & $12-15.5$ \\
\hline
\end{tabular}

tion of the menstrual cycle exceeding 15 days and reddish skin without bruising. The patient suffered from these symptoms over a period of 3 months prior to the clinic visit.

Physical, colposcopic and hysteroscopy examinations showed no signs of fibroids, adenomyosis, endometritis, hyperplasia or pelvic infection with normal uterine and urethral structure and negative cervix and uterus biopsies.

The laboratory results showed a normal complete blood count $(\mathrm{CBC})$ but a low hemoglobin level of $10 \mathrm{~g} / \mathrm{dL}$, a partial thromboplastin time (PTT) of $42 \mathrm{~s}$, a prothrombin time (PT) of $27.3 \mathrm{~s}$, a negative urine pregnancy test and no serum beta human chorionic gonadotropin (hCG).

The patient was a mother of three children and did not have any chronic diseases, blood disorders or polycystic ovary syndrome. The patient had no previous history of miscarriage or ectopic pregnancy, and she had a with a normal hormone profile. She suffered from chronic migraine without auras since the age of 28 years and was treated with $100 \mathrm{mg}$ sumatriptan tablets for 4 years, but without response or improvement, she then changed to topiramate $50 \mathrm{mg}$ once daily. Nine months ago, $800 \mathrm{mg}$ feverfew capsules were administered twice daily for 6 months alone, and then the dose was increased to $800 \mathrm{mg}$ three times daily without improvements.

Previously, she did not take any over-the-counter oral contraceptives or herbs except the combination of paracetamol and caffeine $1 \mathrm{~g}$ when needed during feverfew therapy. Feverfew was discontinued, and the doctor prescribed $10 \mathrm{mg}$ medroxyprogesterone tablets for 10 days beginning on the 21 st day of the next menstrual cycle with $190 \mathrm{mg}$ iron sulfate tablets twice daily and multivitamin tablets once daily for 3 months.

After 4 months, a workup at the obstetrics and gynecology clinic was performed. The laboratory results showed a PT of $14.4 \mathrm{~s}$, a PTT of $29 \mathrm{~s}$, a hemoglobin level of $12 \mathrm{~g} / \mathrm{dL}$, and all coagulation profiles completely recovered to normal levels (Table 1).

\section{Discussion}

Feverfew treats migraine headaches through many mechanisms, and the active constituent parthenolide is a potent inhibitor of polymorphonuclear leukocyte granules, serotonin 5-hydroxytryptamine (5-HT) receptors and prostaglandin synthetase [5]. The dose of feverfew for migraine headaches is 100 to $300 \mathrm{mg}$ up to four times daily for 16 weeks from the standard preparation (0.2-0.4\% parthenolide) and $6.25 \mathrm{mg}$, three times daily, for up to 16 weeks from feverfew supplements with $\mathrm{CO}_{2}$ extracted [5].

However, the adult dose of parthenolide was $0.2-0.6 \mathrm{mg}$ for migraine prophylaxis. According to clinical trials, the ben- eficial effects of Tanacetum parthenium for migraine prophylaxis can be seen within 4 - 6 weeks of initiating therapy. The duration of treatment will vary for individual migraine sufferers [8].

In 2004, a systemic review published in the Cochrane database assessed the evidence against the efficacy of feverfew for preventing migraine and found that the efficacy of feverfew could not be established [1].

Moreover, we used the Naranjo algorithm. It is the most commonly used tool to assess adverse drug reaction causality. This algorithm has ten simple questions. The questions involve the following areas: the temporal relationship, the pattern of response, dechallenge or administration of an antagonist, rechallenge, alternative causes, placebo response, drug level in the body fluids or tissue, dose-response relationship, previous patient experience with the drug, and confirmation by any other objective evidence. The answer to each question is then assigned a score. A score of 9 or greater means that an adverse drug reaction (ADR) is highly probable, a score of 5 to 8 means that an ADR is probable, a score of 1 to 4 means that an ADR is possible, and a score of zero or less means that an ADR is unlikely $[4,13]$. We applied this algorithm in our case. The causality rating for feverfew fell within the range of $5-8$, which indicates a probable ADR. A probable ADR means the event had a plausible time relationship with feverfew intake, and the response to withdrawal (dechallenge) was clinically reasonable with an unknown rechallenge (Table 2).

In our case, a positive dechallenge refers to the adverse drug reaction disappearing after stopping feverfew use, and the patient coagulation profile was improved after 4 months. According to the patient history, physical examination and laboratory investigations, we excluded all possible and alternative causes that could induce vaginal bleeding, e.g., pelvic infections, endometritis and hyperplasia or abnormalities in uterine structure.

In addition, the patient did not take any hormone replacement therapy or oral contraceptives, and these medications can alter coagulation factors. However, a study showed that factor VII and X levels and fibrinogen were significantly increased in patients treated with combined oral contraceptive pills, where the extent of elevation varied depending on the estrogen-progesterone components [14].

During feverfew therapy, the patient took only a combination tablet of paracetamol with caffeine. Paracetamol has scarce inhibition of peripheral cyclooxygenase; it is only a weak inhibitor of platelet aggregation and does not alter the bleeding time [15]. One study did not find an effect of abstinence from caffeine on blood clot lysis time, whereas the effects of caffeine intake on platelet activity are more variable [16].

The vaginal bleeding induced by feverfew in this case 
Table 2. The Naranjo Adverse Drug Reaction Probability Scale

\begin{tabular}{|c|c|c|c|c|}
\hline The Naranjo adverse drug reaction probability scale & Yes & No & $\begin{array}{l}\text { Do not } \\
\text { know }\end{array}$ & Scale \\
\hline Are there previous conclusive reports on this reaction? & +1 & 0 & 0 & 0 \\
\hline Did the adverse reaction improve when the drug was discontinued, or a specific antagonist was administered? & +1 & 0 & 0 & 1 \\
\hline Did the adverse reaction reappear when the drug was readministered? & +2 & -1 & 0 & 0 \\
\hline Was the blood detected in the blood (or other fluids) in concentrations known to be toxic? & +1 & 0 & 0 & 0 \\
\hline Was the reaction more severe when the dose was increased or less severe when the dose was decreased? & +1 & 0 & 0 & 1 \\
\hline Did the patient have a similar reaction to the same or similar drugs in any previous exposure? & +1 & 0 & 0 & 0 \\
\hline Was the adverse event confirmed by any objective evidence? & +1 & 0 & 0 & 1 \\
\hline
\end{tabular}

may be related to inhibiting ADP, thrombin, and the collageninduced aggregation of platelets by interfering with cellular phospholipases, preventing the release of arachidonic acid and inhibiting the release of serotonin from platelets $[3,8]$. Feverfew should be used cautiously by patients planning elective surgery or taking parenteral or oral anticoagulants $[3,12]$.

In our case, the patient received feverfew $800 \mathrm{mg}$ twice daily for 6 months and then increased to $800 \mathrm{mg}$ three times daily. The severity of vaginal bleeding may be related to an increase in the frequency of feverfew and the prolonged duration of use. The treating doctor prescribed medroxyprogesterone to control bleeding and corrected hemoglobin levels with iron supplementation.

\section{Conclusions}

The efficacy of feverfew for preventing migraine has still not been established. Any patient on Tanacetum parthenium should be monitored for blood coagulation factors, and it should be used cautiously by patients planning elective surgery, taking antithrombotic drugs or having coagulant disease. Patients should be advised to taper then discontinue feverfew completely at least 2 - 3 weeks before surgery. The Naranjo scale indicates that a probable relationship exists between feverfew, vaginal bleeding and alterations of coagulation test results.

\section{Acknowledgments}

The authors would like to thank Dr. Tariq Abdulkareem AlRashdan, the obstetrics/gynecology consultant at King Khalid Hospital, Tabuk, Saudi Arabia.

\section{Financial Disclosure}

None to declare.

\section{Conflict of Interest}

The authors declare that there is no conflict of interest related to the manuscript, including commercial, personal, political, and intellectual aspects.

\section{Informed Consent}

The patient's informed consent for publication of this case report was obtained.

\section{Author Contributions}

All authors shared the entire content of the manuscript including data collection, discussion, writing and editing assistance.

\section{Data Availability}

The authors declare that data supporting this study's findings are available within the case report.

\section{References}

1. Wider B, Pittler MH, Ernst E. Feverfew for preventing migraine. Cochrane Database Syst Rev. 2015;4:CD002286.

2. Gruenwald J, Brendler T, Jaenicke C. PDR for herbal medicines: Thomson Reuters. 2007.

3. Kemper KJ. Feverfew (Tanacetum parthenium). 2007.

4. Baranov D. Everything you need to know about feverfew and migraines. Prima. 1999.

5. Pareek A, Suthar M, Rathore GS, Bansal V. Feverfew (Tanacetum parthenium L.): A systematic review. Pharmacogn Rev. 2011;5(9):103-110.

6. Setty AR, Sigal LH. Herbal medications commonly 
used in the practice of rheumatology: mechanisms of action, efficacy, and side effects. Semin Arthritis Rheum. 2005;34(6):773-784.

7. Cardenas J, Reyes-Perez V, Hernandez-Navarro MD, Dorantes-Barron AM, Almazan S, Estrada-Reyes R. Anxiolytic- and antidepressant-like effects of an aqueous extract of Tanacetum parthenium L. Schultz-Bip (Asteraceae) in mice. J Ethnopharmacol. 2017;200:22-30.

8. Asiri YA. The ABC clinical guide to herbs. King Faisal Specialist Hospital \& Research Centre. 2004.

9. Clocon JO, Clocon DG, Galindo DJJG. Botanicals can affect surgical outcomes and follow-up. 2004:59.

10. Baldwin C, Anderson L, Phillipson JJPJ. What pharmacists should know about ginseng? 1986;237:583-586.

11. Pugh WJ, Sambo K. Prostaglandin synthetase inhibitors in feverfew. J Pharm Pharmacol. 1988;40(10):743-745.

12. Berman BM. Complementary medicine and medical edu- cation. BMJ. 2001;322(7279):121-122.

13. Doherty MJ. Algorithms for assessing the probability of an adverse drug reaction. Respir Med CME. 2009;2:6367.

14. Norris LA, Bonnar J. The effect of oestrogen dose and progestogen type on haemostatic changes in women taking low dose oral contraceptives. Br J Obstet Gynaecol. 1996;103(3):261-267.

15. Pini LA, Guerzoni S, Cainazzo M, Ciccarese M, Prudenzano MP, Livrea P. Comparison of tolerability and efficacy of a combination of paracetamol + caffeine and sumatriptan in the treatment of migraine attack: a randomized, double-blind, double-dummy, cross-over study. J Headache Pain. 2012;13(8):669-675.

16. Lee KW, Lip GY. Effects of lifestyle on hemostasis, fibrinolysis, and platelet reactivity: a systematic review. Arch Intern Med. 2003;163(19):2368-2392. 